\title{
FUCHSIAN GROUPS GENERATED BY HALF-TURNS AND GEOMETRICAL CHARACTERIZATION OF HYPERELLIPTIC AND SYMMETRIC RIEMANN SURFACES
}

\author{
J. J. ETAYO and E. MARTÍNEZ*
}

\begin{abstract}
We construct a special type of fundamental regions for any Fuchsian group $F$ generated by an even number of half-turns, and for certain non-Euclidean crystallographic groups (NEC groups in short). By comparing these regions we give geometrical conditions in order to $F$ be the canonical Fuchsian subgroup of one of those NEC groups. Precisely speaking, we deal with NEC groups of algebraic genus 0 having all periods in the signature equal to 2 . By means of these conditions we give a characterization of hyperelliptic and symmetric Riemann surfaces.
\end{abstract}

\section{Introduction}

Hyperelliptic Riemann surfaces constitute a very important class of surfaces. They are studied since XIX century [11]. There exist several geometrical characterizations of these surfaces, for instance, [6], [8]. A different way to characterize hyperelliptic Riemann surfaces is by means of Fuchsian groups. Let $S=\mathscr{D} / F^{*}$ be a Riemann surface of genus $p \geq 2 ; S$ is hyperelliptic if and only if there exists a Fuchsian group $F$ containing $F^{*}$ as a subgroup of index 2 , with signature $\left(0 ;+;\left[2^{2 p+2}\right],\{-\}\right)$.

Analogously for a Klein surface we have a characterization of hyperellipticity in terms of NEC groups. Let $X=\mathscr{D} / \Gamma^{*}$ be a Klein surface where $\Gamma^{*}$ has signature $\left(g ; \pm ;[-],\left\{(-)^{k}\right\}\right)$. The surface $X$ is hyperelliptic if and only if there exists an NEC group $\Gamma$ containing $\Gamma^{*}$ as a subgroup of index 2, [2]. In such a case $\Gamma$ has algebraic genus 0 and each proper period and link-period in its signature is 2 .

A Riemann surface $S$ is said to be symmetric if and only if $S$ admits an anticonformal automorphism $\psi$ such that $\psi^{2}$ is the identity. Let us observe that the quotient $X=S /\langle\psi\rangle$ is a Klein surface. If the Riemann surface $S$ is

\footnotetext{
* The first author is partially supported by BFM2002-04797 and HPRN-CT-2001-0271 and the second one by BFM2002-04801.

Received September 30, 2002.
} 
both hyperelliptic and symmetric then $X$ is a hyperelliptic Klein surface [4] and in this case we have the following diagram relating the different groups.

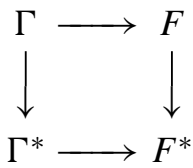

Fuchsian groups with all periods equal to 2 are generated by half-turns, that is elliptic elements with trace 0 . We are interested in the following geometrical problem that allows us to give a characterization of hyperelliptic and symmetric Riemann surfaces. Given a Fuchsian group $F$ generated by half-turns, what conditions must $F$ satisfy in order to be the canonical Fuchsian subgroup of an NEC group $\Gamma$, that is to say, in what conditions $F=\Gamma^{+}$? Obviously the group $\Gamma$ has then each period equal to 2 in its signature. We will give the answer by means of geometrical conditions which fundamental regions of the groups $F$ and $\Gamma^{+}$must satisfy.

For it, we need to compare regions of both groups. Canonical regions are not adequate because of the different number of sides of the regions of $F$ and $\Gamma^{+}$. Instead of them, we construct a special type of fundamental regions (hyperbolic polygons whose angles satisfy some conditions) for these groups. Given such a fundamental region of $F$ we may say when $F=\Gamma^{+}$and in this case the class of NEC groups to which $\Gamma$ belongs.

The work is organized as follows. In Section 2 we give the necessary preliminaries about Fuchsian and NEC groups and canonical fundamental regions. In Section 3 we construct a new fundamental region for concerned Fuchsian group $F$ and NEC group $\Gamma$. Then, in Section 4, we compare the obtained regions and give the geometrical conditions in order to $F$ be the canonical Fuchsian subgroup of $\Gamma$. Finally, in Section 5, we rewrite the results in terms of hyperelliptic Riemann surfaces.

\section{Preliminaries}

An NEC group $\Gamma$ is a discrete subgroup of isometries of the hyperbolic plane $\mathscr{D}$ (including orientation-reversing isometries) with compact quotient $\mathscr{D} / \Gamma$ [10]. The signature of $\Gamma$ is the following symbol:

$$
\sigma(\Gamma):\left(g, \pm,\left[m_{1}, \ldots, m_{r}\right],\left\{\left(n_{i, 1}, \ldots, n_{i, s_{i}}\right)_{i=1, \ldots, k}\right\}\right),
$$

where $g, k \geq 0, m_{i}, n_{i, j} \geq 2$ and every number is an integer. The signature determines the algebraic structure of $\Gamma$ [7]. The quotient $X=\mathscr{D} / \Gamma$ has topological genus $g$ and $k$ boundary components. $X$ is orientable if the sign in (1) is ' + ' and nonorientable otherwise. The brackets $\left(n_{i, 1}, \ldots, n_{i, s_{i}}\right)$ are called 
cycle-periods and the numbers $m_{i}$ and $n_{i, j}$ are called proper periods and link periods, respectively. If $r=0, k=0$ or $s_{i}=0$, we write in each respective case [-], $\{-\},(-)$. Also we write $m_{i}^{t}$ or $n_{i, j}^{t}$ when a period is repeated $t$ times.

The algebraic genus of $\Gamma$ is $p=\eta g+k-1$ where $\eta=2$ or 1 according to the sign in $\sigma$ be ' + ' or ' - '. The area of $\Gamma$ is the area of any fundamental region of $\Gamma$. It is denoted by $|\Gamma|$ and it satisfies

$$
|\Gamma|=2 \pi\left(\eta g+k-2+\sum_{i=1}^{r}\left(1-\frac{1}{m_{i}}\right)+\frac{1}{2} \sum_{i=1}^{k} \sum_{j=1}^{s_{i}}\left(1-\frac{1}{n_{i, j}}\right)\right) .
$$

An NEC group $\Gamma$ with signature as (1) exists if and only if $|\Gamma|>0$.

Let $\Gamma$ be an NEC group with signature as (1). $\Gamma$ is generated by $x_{i},(i=$ $1, \ldots, r)$ elliptic transformations, $e_{i},(i=1, \ldots, k)$ hyperbolic transformations, $c_{i, j},\left(i=1, \ldots, k ; j=0,1, \ldots, s_{i}\right)$ reflections and $a_{i}, b_{i},(i=$ $1, \ldots, g)$ hyperbolic transformations (if sign is ' + ') or $d_{i},(i=1, \ldots, g)$ glide reflections (if sign is '-'). The generators satisfy the following relations:

$$
\begin{array}{ll}
x_{i}^{m_{i}}=1 & i=1, \ldots, r, \\
c_{i, j-1}^{2}=c_{i, j}^{2}=\left(c_{i, j-1} \cdot c_{i, j}\right)^{n_{i, j}}=1 & i=1, \ldots, k, j=1, \ldots, s_{i}, \\
e_{i}^{-1} \cdot c_{i, 0} \cdot e_{i} \cdot c_{i, s_{i}}=1 & i=1, \ldots, k,
\end{array}
$$

and

$$
\begin{array}{ll}
\prod_{i=1}^{r} x_{i} \cdot \prod_{i=1}^{k} e_{i} \cdot \prod_{i=1}^{g}\left[a_{i}, b_{i}\right]=1 & \text { if sign ' }+, \\
\prod_{i=1}^{r} x_{i} \cdot \prod_{i=1}^{k} e_{i} \cdot \prod_{i=1}^{g} d_{i}^{2}=1 & \text { if sign '-', }
\end{array}
$$

where $\left[a_{i}, b_{i}\right]$ denotes the commutator $a_{i} b_{i} a_{i}^{-1} b_{i}^{-1}$.

An NEC group $\Gamma$ with sign ' + ' in the signature and $k=0$, (hence $g \geq 2$ ) is a surface Fuchsian group and in this case $X=\mathscr{D} / \Gamma$ is a Riemann surface. An NEC group which is not a Fuchsian group is called a proper NEC group.

The subgroup of orientation preserving elements of a proper NEC group $\Gamma$ is called the canonical Fuchsian subgroup and it is denoted by $\Gamma^{+}$. If $\Gamma$ has a signature as (1) then $\Gamma^{+}$has the following signature $\sigma\left(\Gamma^{+}\right)$, [9]:

$$
\begin{aligned}
\left(p,+,\left[m_{1}, m_{1}, m_{2}, m_{2}, \ldots, m_{r}, m_{r}, n_{1,1},\right.\right. \\
\left.\left.\ldots, n_{1, s_{1}}, \ldots, n_{k, 1}, \ldots, n_{k, s_{k}}\right],\{-\}\right) .
\end{aligned}
$$

As an immediate consequence we have the following Proposition for later convenience, 
Proposition 2.1. Let $F$ be a Fuchsian group with signature

$$
\left(0,+,\left[2^{2 r}\right],\{-\}\right),
$$

and let us suppose that $F=\Gamma^{+}$for an NEC group $\Gamma$. Then the signature of $\Gamma$ is one of the following signatures:

1) $\sigma\left(\Gamma_{1}\right):\left(0,+,[-],\left(\left\{\left(2^{2 r}\right)\right\}\right)\right.$.

2) $\sigma\left(\Gamma_{2}\right):\left(0,+,\left[2^{q}\right],\left(\left\{\left(2^{s}\right)\right\}\right)\right.$, where $2 q+s=2 r$.

3) $\sigma\left(\Gamma_{3}\right):\left(0,+,\left[2^{r}\right],(\{(-)\})\right.$.

4) $\sigma\left(\Gamma_{4}\right):\left(1,-,\left[2^{r}\right],(\{-\})\right.$.

Although 1) and 3) can be considered as particular cases of 2) for technical reasons it is better to study them as separated cases.

Wilkie in [10] found a class of fundamental region $W$ from what he obtained the algebraic structure of NEC groups. These regions are called canonical regions and also Wilkie regions.

For an NEC group $\Gamma$ with signature as (1) the region $W$ is a hyperbolic polygon with sides labelled in anticlockwise order as follows

$$
\underbrace{\ldots \xi_{i}, \xi_{i}^{\prime}, \ldots}_{i=1, \ldots, r} ; \underbrace{\ldots \varepsilon_{i}, \gamma_{i, 0}, \gamma_{i, 1}, \ldots, \gamma_{i, s_{i}}, \varepsilon_{i}^{\prime}, \ldots}_{i=1, \ldots, k} ; \underbrace{\ldots \alpha_{i}, \beta_{i}^{\prime}, \alpha_{i}^{\prime}, \beta_{i}, \ldots}_{i=1, \ldots, g},
$$

if the sign is ' + ' or

$$
\underbrace{\ldots \xi_{i}, \xi_{i}^{\prime}, \ldots}_{i=1, \ldots r} ; \underbrace{\ldots \varepsilon_{i}, \gamma_{i, 0}, \gamma_{i, 1}, \ldots, \gamma_{i, s_{i}}, \varepsilon_{i}^{\prime}, \ldots}_{i=1, \ldots, k} ; \underbrace{\ldots \delta_{i}, \delta_{i}^{*}, \ldots}_{i=1, \ldots, g}
$$

if the sign is ' - ', where

$$
\begin{array}{lll}
x_{i}\left(\xi_{i}^{\prime}\right)=\xi_{i}, & e_{i}\left(\varepsilon_{i}^{\prime}\right)=\varepsilon_{i}, & c_{i, j}\left(\gamma_{i, j}\right)=\gamma_{i, j}, \\
a_{i}\left(\alpha_{i}^{\prime}\right)=\alpha_{i}, & b_{i}\left(\beta_{i}^{\prime}\right)=\beta_{i}, & d_{i}\left(\delta_{i}^{*}\right)=\delta_{i} .
\end{array}
$$

Let us denote by $\left\langle s_{1}, s_{2}\right\rangle$ the angle between two consecutive sides. In the region $W$ we have

$$
\left\langle\varepsilon_{i}, \gamma_{i, 0}\right\rangle+\left\langle\gamma_{i, s_{i}}, \varepsilon_{i}^{\prime}\right\rangle=\pi
$$

and the sum of the remaining angles (accidental cycle) is $2 \pi$. Without loss of generality we may suppose that $W$ is a convex polygon and

$$
\left\langle\varepsilon_{i}, \gamma_{i, 0}\right\rangle=\left\langle\gamma_{i, s_{i}}, \varepsilon_{i}^{\prime}\right\rangle=\pi / 2
$$




\section{Regions}

Through this section we deal with fundamental regions of groups $F, \Gamma_{1}, \Gamma_{2}, \Gamma_{3}$ and $\Gamma_{4}$, as in Proposition 2.1. For $F, \Gamma_{2}, \Gamma_{3}$ and $\Gamma_{4}$ (the canonical region of $\Gamma_{1}$ is different) we start with a canonical fundamental region $W_{1}$ and we shall obtain a new fundamental region by means of a cutting and pasting procedure. This procedure is common for the four groups except the last steps.

Let $W_{1}$ be the following canonical region for the group $G=F, \Gamma_{2}, \Gamma_{3}, \Gamma_{4}$, with vertices labelled by capital letters and described in an anticlockwise order.

$$
\xi_{1}, X_{1}, \xi_{1}^{\prime}, P_{1}, \ldots, \xi_{\tau}, X_{\tau}, \xi_{\tau}^{\prime}, P_{\tau}, \mathscr{L}, Q
$$

where $\mathscr{L}$ is

- $=\emptyset$, if $G=F$.

- $=\varepsilon, \gamma_{0}, \gamma_{1}, \ldots, \gamma_{s}, \varepsilon^{\prime}$, if $G=\Gamma_{2}$, and then $Q=P_{\tau+1}$.

- $=\varepsilon, \gamma, \varepsilon^{\prime}$, if $G=\Gamma_{3}$, and then $Q=P_{\tau+1}$.

- $=\delta, P_{\tau+1}, \delta^{*}$, if $G=\Gamma_{4}$, and $Q=P_{\tau+2}$.

In the second and third cases it is not necessary to write explicitly the intermediate vertices because the angles at these vertices are $\frac{\pi}{2}$.

In the above description, points $X_{i}$ are the fixed points of elliptic transformations, $x_{i}$, of order 2. The angles at vertices $P_{i}$ are $\theta_{i}$ and $\sum_{i} \theta_{i}=2 \pi$.

Let us observe that $\tau=2 r, q, r, r$ in the four respective cases.

Let $\eta_{i}$ be the hyperbolic segment between $X_{i}$ and $X_{i+1}$. In the step $i$ we cut by $\eta_{i}$ the region $W_{i}$, which becomes divided in two subpolygons: one of them, say $R_{i}$, must contain the side $\xi_{i+1}$. Let $W_{i+1}$ be the new region

$$
W_{i+1}=\left(W_{i}-R_{i}\right) \cup x_{i+1}\left(R_{i}\right)
$$

obtained identifying $\xi_{i+1} \rightarrow \xi_{i+1}^{\prime}$ by means of $x_{i+1}^{-1}=x_{i+1}$.

In the first step we obtain

$$
W_{2}:=\xi_{1}, X_{1}, \eta_{1}, X_{2}, x_{2}\left(\eta_{1}\right), x_{2}\left(X_{1}\right), x_{2}\left(\xi_{1}^{\prime}\right), P_{2}, Z
$$

where $Z$ denotes the remaining sides. Let us observe that the angle $\pi$ in $X_{1}$ becomes divided in two angles: the interior angle $\alpha_{1}$ between $\xi_{1}$ and $\eta_{1}$, and the angle $\pi-\alpha_{1}$. Furthermore, the angle in the vertex $P_{2}$ is now $\theta_{1}+\theta_{2}$. The next step produces

$$
\begin{aligned}
W_{3}:=\xi_{1}, X_{1}, \eta_{1}, X_{2}, \eta_{2}, X_{3}, & x_{3}\left(\eta_{2}\right), x_{3}\left(X_{2}\right), \\
& x_{3} \cdot x_{2}\left(\eta_{1}\right), x_{3} \cdot x_{2}\left(X_{1}\right), x_{3} \cdot x_{2}\left(\xi_{1}^{\prime}\right), P_{3}, Z
\end{aligned}
$$


Now we denote the interior angle between $\eta_{1}$ and $\eta_{2}$ by $\alpha_{2}$. The angle in $P_{3}$ is now $\theta_{1}+\theta_{2}+\theta_{3}$.

Repeating the procedure we arrive to the following polygon:

$\xi_{1}, X_{1}, \eta_{1}, X_{2}, \eta_{2}, X_{3}, \eta_{3}, \ldots, \eta_{\tau-1}, X_{\tau}, x_{\tau}\left(\eta_{\tau-1}\right), x_{\tau}\left(X_{\tau-1}\right), x_{\tau} \cdot x_{\tau-1}\left(\eta_{\tau-2}\right)$,

$x_{\tau} \cdot x_{\tau-1}\left(X_{\tau-2}\right), \ldots, x_{\tau} \cdot x_{\tau-1} \cdot \ldots \cdot x_{2}\left(\eta_{1}\right), x_{\tau} \cdot x_{\tau-1} \cdot \ldots \cdot x_{2}\left(X_{1}\right), P_{\tau}, Z$

where the angles at the vertices $X_{i}$ are $\alpha_{i}$ and in the respective images are $\pi-\alpha_{i}$.

In this point we must split our study in four cases according to $G$ be $F, \Gamma_{2}, \Gamma_{3}$ or $\Gamma_{4}$.

- Case $G=F$.

The angle in $P_{\tau}=\theta_{1}+\theta_{2}+\cdots+\theta_{n}=2 \pi$, and $P_{\tau}$ becomes an interior point. Moreover

$$
\begin{gathered}
x_{\tau} \cdot x_{\tau-1} \cdot \ldots \cdot x_{2}\left(X_{1}\right)=x_{1}\left(X_{1}\right)=X_{1}, \\
x_{\tau} \cdot x_{\tau-1} \cdot \ldots \cdot x_{2}\left(\xi_{1}^{\prime}\right)=x_{1}\left(\xi_{1}^{\prime}\right)=\xi_{1},
\end{gathered}
$$

and the angle in $X_{1}$ is $\pi$. We have the following polygon $W_{F}$, drawn for $\tau=6$.

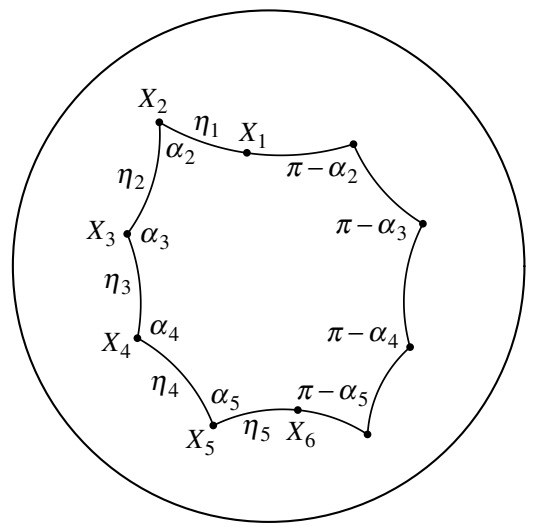

FIGURE 1. Region $W_{F}$ for $\tau=6$

- Case $G=\Gamma_{2}$.

Let $\lambda$ be the perpendicular segment from $X_{\tau}$ to $\gamma_{0}$, dividing $\gamma_{0}$ in $\widehat{\gamma}_{0}$ and $\bar{\gamma}_{0}$. Cut by $\lambda$ the subpolygon that contains the side $\varepsilon$ and glue $\varepsilon \rightarrow \varepsilon^{\prime}$ by means of $e^{-1}$. The angle in $P_{\tau+1}$ is now $2 \pi$ and

$$
\begin{gathered}
e^{-1} \cdot x_{\tau} \cdot x_{\tau-1} \cdots x_{2}\left(X_{1}\right)=x_{1}\left(X_{1}\right)=X_{1}, \\
e^{-1} \cdot x_{\tau} \cdot x_{\tau-1} \cdots x_{2}\left(\xi_{1}^{\prime}\right)=x_{1}\left(\xi_{1}^{\prime}\right)=\xi_{1} .
\end{gathered}
$$


- Case $G=\Gamma_{3}$.

The procedure is similar to the previous case, $\lambda$ being now the perpendicular segment from $X_{\tau}$ to $\gamma$ dividing it in $\widehat{\gamma}$ and $\bar{\gamma}$.

We obtain the respective polygons $W_{\Gamma_{2}}$ and $W_{\Gamma_{3}}$. They are shown for values $\tau=4, s=2$ in Figure 2(a) and (b).

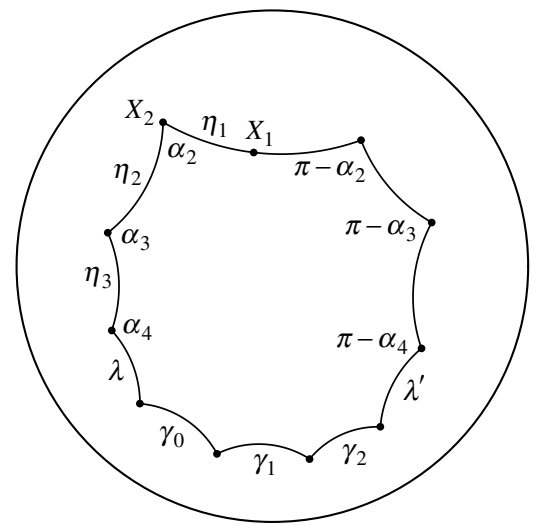

(a) Region $W_{\Gamma_{2}}$ for $\tau=4$ and $s=2$

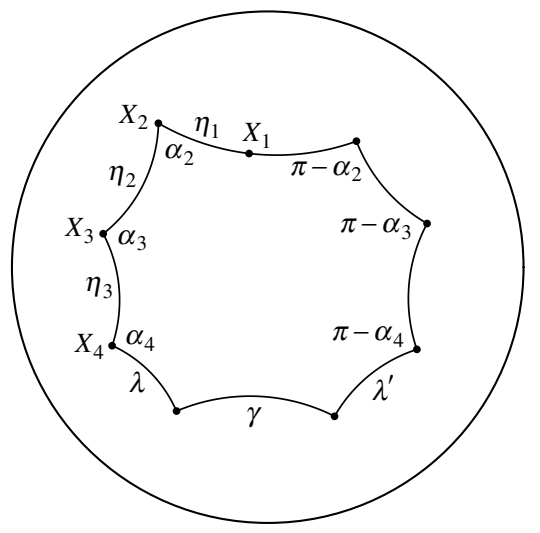

(b) Region $W_{\Gamma_{3}}$ for $\tau=4$

FIGURE 2

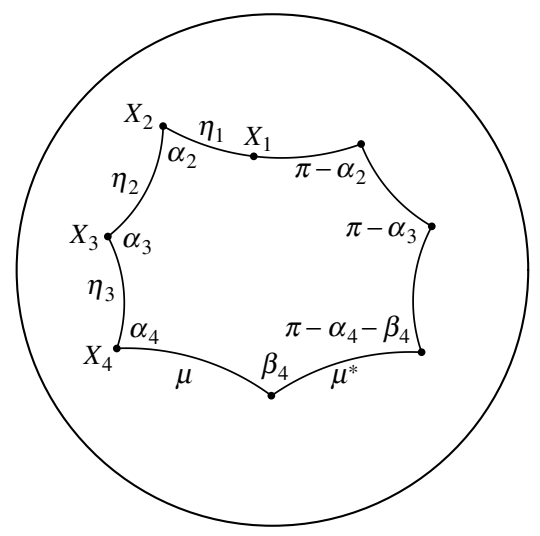

Figure 3. Region $W_{\Gamma_{4}}$ for $\tau=4$

- Case $G=\Gamma_{4}$.

We need two steps in this case. First, let $\lambda$ be the segment from $X_{\tau+1}$ to $P_{\tau+1}$. Cut by $\lambda$ the subpolygon which contains the side $\delta$ and glue $\delta \rightarrow \delta^{*}$ by means of $d^{-1}$. We obtain a new region. 
Now let $\mu$ be the segment between $X_{\tau}$ and $d^{-1}\left(X_{\tau}\right)$ and cut by $\mu$ the subpolygon that contains the side $\lambda$. Glue $\lambda \rightarrow d^{-1}(\lambda)$ by means of $d^{-1}$. We finally obtain the region $W_{\Gamma_{4}}$, shown in Figure 3 for $\tau=4$.

We have proved the following result:

Proposition 3.1. The above regions $W_{F}, W_{\Gamma_{2}}, W_{\Gamma_{3}}$ and $W_{\Gamma_{4}}$ are, respectively, fundamental regions of groups $F, \Gamma_{2}, \Gamma_{3}$ and $\Gamma_{4}$.

Let $\mathscr{P}_{F}, \mathscr{P}_{2}, \mathscr{P}_{3}, \mathscr{P}_{4}$, be the classes of marked polygons:

- $\mathscr{P}_{F}$ :

$$
X_{2}, \eta_{2}, \ldots, X_{\tau-1}, \eta_{\tau-1}, X_{\tau}, \eta_{\tau-1}^{\prime}, X_{\tau-1}^{\prime}, \ldots, \eta_{2}^{\prime}, X_{2}^{\prime}, \eta_{1}^{\prime}, X_{1}, \eta_{1},
$$

where $\widehat{X_{1}}=\widehat{X_{\tau}}=\pi, \widehat{X}_{i}+\widehat{X}_{i}^{\prime}=\pi, i=2, \ldots, \tau-1$.

- $\mathscr{P}_{2}$ :

$$
\begin{aligned}
& X_{2}, \eta_{2}, \ldots, X_{\tau-1}, \eta_{\tau-1}, X_{\tau}, \lambda, \gamma_{0}, \gamma_{1}, \ldots, \gamma_{s}, \\
& \lambda^{\prime}, X_{\tau}^{\prime}, \ldots, \eta_{2}^{\prime}, X_{2}^{\prime}, \eta_{1}^{\prime}, X_{1}, \eta_{1},
\end{aligned}
$$

where $\widehat{X_{1}}=\pi, \widehat{X}_{i}+\widehat{X}_{i}^{\prime}=\pi, i=2, \ldots, \tau,\left\langle\lambda, \gamma_{0}\right\rangle=\left\langle\gamma_{0}, \gamma_{1}\right\rangle=\cdots=$ $\left\langle\gamma_{s-1}, \gamma_{s}\right\rangle=\left\langle\gamma_{s}, \lambda^{\prime}\right\rangle=\frac{\pi}{2}$

- $\mathscr{P}_{3}$ :

$$
X_{2}, \eta_{2}, \ldots, X_{\tau-1}, \eta_{\tau-1}, X_{\tau}, \lambda, \gamma, \lambda^{\prime}, X_{\tau}^{\prime}, \ldots, \eta_{2}^{\prime}, X_{2}^{\prime}, \eta_{1}^{\prime}, X_{1}, \eta_{1},
$$

where $\widehat{X_{1}}=\pi, \widehat{X}_{i}+\widehat{X}_{i}^{\prime}=\pi, i=2, \ldots, \tau,\langle\lambda, \gamma\rangle=\left\langle\gamma, \lambda^{\prime}\right\rangle=\frac{\pi}{2}$.

- $\mathscr{P}_{4}$ :

$$
X_{2}, \eta_{2}, \ldots, X_{\tau-1}, \eta_{\tau-1}, X_{\tau}, \mu, X_{\tau}^{\prime \prime}, \mu^{*}, X_{\tau}^{\prime}, \ldots, \eta_{2}^{\prime}, X_{2}^{\prime}, \eta_{1}^{\prime}, X_{1}, \eta_{1},
$$$$
\text { where } \widehat{X_{1}}=\pi, \widehat{X}_{i}+\widehat{X}_{i}^{\prime}=\pi, i=2, \ldots, \tau-1, \widehat{X_{\tau}}+\widehat{X_{\tau}^{\prime}}+\widehat{X_{\tau}^{\prime \prime}}=\pi .
$$

The next result is the converse of Proposition 3.1.

Proposition 3.2. Every polygon $W \in \mathscr{P}_{F}, \mathscr{P}_{2}, \mathscr{P}_{3}, \mathscr{P}_{4}$ is a fundamental region of a Fuchsian group $F$ or NEC group $\Gamma_{2}, \Gamma_{3}, \Gamma_{4}$, respectively, where the signatures of these groups are as in Proposition 2.1.

Proof. We must distinguish according to whether $W$ belongs to $\mathscr{P}_{F}, \mathscr{P}_{2}$, $\mathscr{P}_{3}, \mathscr{P}_{4}$.

- $W \in \mathscr{P}_{F}$.

Let us observe that the geodesics containing the sides $\eta_{i}, \eta_{i}^{\prime}, i=2, \ldots, \tau-$ 1 , do not intersect. Thus there exist hyperbolic transformations $f_{i}$ which map 
$\eta_{i}^{\prime}$ on $\eta_{i}$ such that $f_{i}\left(X_{i-1}^{\prime}\right)=X_{i-1}$ and $f_{i}\left(X_{i}^{\prime}\right)=X_{i}$. Let $x_{1}$ and $x_{\tau}$ be the elliptic transformations of order two and fixed points $X_{1}$ and $X_{\tau}$, respectively.

The segment between $X_{1}$ and $X_{\tau}$ divides $W$ in two parts. Let $P$ be an interior point of the right part. We draw the segments $l_{1}, l_{2}, l_{3}, \ldots, l_{\tau-1}, l_{\tau}$, from $P$ to $X_{1}, X_{2}^{\prime}, X_{3}^{\prime}, \ldots, X_{\tau-1}^{\prime}, X_{\tau}$.

We cut the triangle $T_{1}=P X_{1} X_{2}^{\prime}$ and take $x_{1}\left(T_{1}\right)$. We have obtained a new region $W_{1}=\left(W-T_{1}\right) \cup x_{1}\left(T_{1}\right)$. Let us now consider the triangle $T_{2}=$ $P X_{2}^{\prime} X_{3}^{\prime}$. Cut $T_{2}$ and take $f_{2}\left(T_{2}\right)$. We have a new region $W_{2}=\left(W-\left(T_{1} \cup T_{2}\right)\right)$ $\cup x_{1}\left(T_{1}\right) \cup f_{2}\left(T_{2}\right)$. Repeating the process for the triangles $T_{i}=P X_{i}^{\prime} X_{i+1}^{\prime}$, $i=3, \ldots, \tau-2$, we obtain a region

$$
W_{\tau-1}=\left(W-\bigcup_{i} T_{i}\right) \cup x_{1}\left(T_{1}\right) \cup \bigcup_{i} f_{i}\left(T_{i}\right) .
$$

For the last step we cut the triangle $T_{\tau-1}=P X_{\tau-1}^{\prime} X_{\tau}$ and take $x_{\tau}\left(T_{\tau-1}\right)$. We form the region $W_{\tau}$, whose perimeter is

$$
P, l_{1}, X_{1}, x_{1}\left(l_{1}\right), x_{1}(P) \equiv f_{2}\left(X_{2}^{\prime}\right), f_{2}\left(l_{2}\right), \ldots, x_{\tau}\left(l_{\tau}\right), X_{\tau}, l_{\tau} .
$$

Let us observe that $\widehat{X}_{i}=\pi, i=2, \ldots, \tau-2$. Moreover, $f_{\tau-1} \cdot x_{\tau}\left(x_{\tau}\left(l_{\tau-1}\right)\right)=$ $f_{\tau-1}\left(l_{\tau-1}\right)$ and $f_{\tau-1} \cdot x_{\tau}\left(X_{\tau-1}\right)=X_{\tau-1}$. Hence $f_{\tau-1} \cdot x_{\tau}$ is an elliptic transformation of order two with fixed point $X_{\tau-1}$. Call $x_{\tau-1}=f_{\tau-1} \cdot x_{\tau}$. Analogously, $x_{i}=f_{i} \cdot f_{i+1}^{-1}, i=2, \ldots, \tau-2$, are elliptic transformations of order two with fixed point $X_{i}$. Furthermore, the sum of the angles at the images of the point $P$ is $2 \pi$.

We have obtained a canonical fundamental region of a Fuchsian group $F$, with signature $\left(0,+,\left[2^{\tau}\right],\{-\}\right)$.

- $W \in \mathscr{P}_{2}$ (respectively $\mathscr{P}_{3}$ ).

As in previous case there exist hyperbolic transformations $f_{i}$ mapping $\eta_{i}^{\prime} \rightarrow$ $\eta_{i}, i=2, \ldots, \tau-1$, and an elliptic transformation $x_{1}$ with fixed point $X_{1}$. Besides we have another hyperbolic transformation $f_{\tau}$ mapping $\lambda^{\prime} \rightarrow \lambda$.

Let $l_{1}, \ldots, l_{\tau}$ be as above and $l_{\tau+1}$ the perpendicular from $P$ to $\gamma_{s}$ (resp. $\gamma$ ) dividing it in $\widehat{\gamma}_{s}$ and $\bar{\gamma}_{s}$ (resp. in $\widehat{\gamma}$ and $\bar{\gamma}$ ).

Repeat the cutting and pasting procedure of the former case for $i=1, \ldots$, $\tau-1$, in the last step, cut the quadrilateral $l_{\tau}, \lambda^{\prime}, \bar{\gamma}_{s}, l_{\tau+1}\left(\right.$ resp. $\left.l_{\tau}, \lambda^{\prime}, \bar{\gamma}, l_{\tau+1}\right)$ and glue $\lambda^{\prime} \rightarrow \lambda$ by means of $f_{\tau}$.

We obtain a canonical fundamental region of an NEC group $\Gamma_{2}$ (resp. $\left.\Gamma_{3}\right)$ with signature $\left(0,+,\left[2^{\tau}\right],\left\{\left(2^{s}\right)\right\}\right)$, or respectively $\left(0,+,\left[2^{\tau}\right],\{(-)\}\right)$.

- $W \in \mathscr{P}_{4}$.

Let now $l_{1}, \ldots, l_{\tau}$, be as in previous cases and let $l_{\tau+1}$ be the segment from $P$ to $X_{\tau}^{\prime \prime}$. There exist an elliptic transformation $x_{1}$, hyperbolic transformations $f_{2}, \ldots, f_{\tau-1}$, and a glide-reflection $d$ which maps $\mu^{*} \rightarrow \mu$. 
Repeating the same procedure we arrive to the last step which consists of cutting the triangle $P X_{\tau}^{\prime} X_{\tau}^{\prime \prime}$ and gluing $\mu^{*} \rightarrow \mu$ by means of $d$. We need an extra cut. Let $l_{\tau+2}$ be the segment from $P$ to $d(P)$. Now we cut the triangle $P X_{\tau}^{\prime \prime} d(P)$ and glue $l_{\tau+1}$ with $d\left(l_{\tau+1}\right)$ by means of $d$. So that we obtain a canonical fundamental region of an NEC group $\Gamma_{4}$ with signature $\left(1,-,\left[2^{\tau}\right],\{-\}\right)$.

Up to now we have studied the groups $F, \Gamma_{2}, \Gamma_{3}, \Gamma_{4}$. Now we deal with the case of the group $\Gamma_{1}$. Let $\mathscr{P}_{1}$ be the class of hyperbolic polygons

$$
\gamma_{1}, \gamma_{2}, \ldots, \gamma_{2 r}
$$

where every angle is $\frac{\pi}{2}$. It is well known that every NEC group $\Gamma_{1}$ has a canonical fundamental region $W \in \mathscr{P}_{1}$ and, conversely, every $W \in \mathscr{P}_{1}$ is the fundamental region of an NEC group with signature $\left(0,+,[-],\left\{\left(2^{2 r}\right)\right\}\right)$.

Now we are going to study a parametrization of $\mathscr{P}_{i}$. In order to construct a hyperbolic polygon with $n$ sides and angles we need $2 n-3$ parameters. When several lengths of sides or some angles are given we must discount them. If the polygon is to be a fundamental region of a Fuchsian or NEC group several cycles of angles may appear, and in these cases we usually know the sum of the angles in each cycle, and then we must discount the number of those cycles.

For our polygons $W \in \mathscr{P}_{i}$ we have,

- $W \in \mathscr{P}_{0}\left(=\mathscr{P}_{F}\right)$ :

The number of free lengths is $\tau-4$, two angles $(=\pi)$ are given, and we have $\tau-2$ cycles with two angles in each one. The number of free parameters is then $2 \tau-6$.

- $W \in \mathscr{P}_{1}$ :

The angles are given. So we have $2 r-3$ free parameters.

- $W \in \mathscr{P}_{2}$ :

The number of free lengths is $\tau+s+1-3$. The polygon has $s+2$ right angles and another one equal to $\pi$. The remaining angles appear in $\tau-1$ two-cycles. The total number of free parameters is $2 \tau+s-3$.

- $W \in \mathscr{P}_{3}$ :

It is a particular case of the previous one when $s=0$. So the number is $2 \tau-3$.

- $W \in \mathscr{P}_{4}$ :

The number of free lengths is now $\tau-3$. An angle equal to $\pi$ is given. The remaining angles appear in $\tau-2$ two-cycles and in one three-cycle. Then the total number is $2 \tau-3$.

We call dimension of a polygon $W$ the number of free parameters appearing in its construction and denote this number by $d(W)$. 
Proposition 3.3. The dimension of each polygon $W^{i} \in \mathscr{P}_{i}, i=0,1, \ldots, 4$, equals the dimension of the Teichmüller space of the groups $F, \Gamma_{1}, \ldots, \Gamma_{4}$.

Proof. According to the different values of $\tau$ (see the signatures of Proposition 2.1), we have

$$
\begin{aligned}
& d\left(W^{0}\right)=2 \tau-6=4 r-6, \\
& d\left(W^{1}\right)=2 r-3, \\
& d\left(W^{2}\right)=2 \tau+s-3=2 q+s-3, \\
& d\left(W^{3}\right)=2 \tau-3=2 r-3, \\
& d\left(W^{4}\right)=2 \tau-3=2 r-3 .
\end{aligned}
$$

And these numbers coincide with the dimension of the Teichmüller spaces of the respective groups.

\section{Comparison of regions}

In this section we study the geometrical conditions that $W_{F}$ must satisfy in order to the group $F$ be the canonical Fuchsian group of an NEC group $\Gamma_{i}$ having a fundamental region of type $W_{\Gamma_{i}}$. For this purpose we must firstly study the regions $W_{\Gamma_{i}^{+}}$, obtained by doubling the region $W_{\Gamma_{i}}$. We shall distinguish the four cases.

- $W_{\Gamma_{1}}$

In this case $W_{\Gamma_{1}} \in \mathscr{P}_{1}$. Let us reflect the region by means of $c_{r}$, the reflection on the side $\gamma_{r}$. We obtain a right-angled region $W_{\Gamma_{1}^{+}}=W_{\Gamma_{1}} \cup c_{r}\left(W_{\Gamma_{1}}\right)$.

$\bullet W_{\Gamma_{2}}$

We describe the procedure to be followed in case $\Gamma_{2}$ for a particular simple case, namely $q=2$ and $s=2$. Let $W_{\Gamma_{2}}$ be the polygon as Figure 4(a).

If we reflect the region by means of $c_{i}$, the reflection on the side $\gamma_{i}$, we do not obtain a region of the type $W_{F}$. Instead of we must reflect different parts of the region by means of different reflections. In a precise way, let us denote by $v_{0}$ and $v_{1}$ the segments from the point $X_{2}$ to the vertices in the angles $\left\langle\gamma_{0}, \gamma_{1}\right\rangle,\left\langle\gamma_{1}, \gamma_{2}\right\rangle$, respectively. The triangles $\lambda, \gamma_{0}, \nu_{0} ; \nu_{0}, \gamma_{1}, \nu_{1}$; are reflected by means of $c_{0}$ and $c_{1}$, respectively. The remaining part of $W_{\Gamma_{2}}$ is reflected by means of $c_{2}$, (Figure 4(b)). Let us observe that the angle between $c_{i}\left(v_{i}\right)$ and $c_{i+1}\left(v_{i}\right)$ is always $\pi$. Let us denote $c_{1} \cdot c_{0}$ as $x_{3}$ and $c_{2} \cdot c_{1}$ as $x_{4}$; and the angles $\left\langle\lambda, v_{0}\right\rangle,\left\langle v_{0}, v_{1}\right\rangle,\left\langle v_{1}, \eta_{1}\right\rangle$ as $\phi_{0}, \phi_{1}$, and $\phi_{2}$, respectively. Obviously $\phi_{0}+\phi_{1}+\phi_{2}=\alpha_{2}$. 


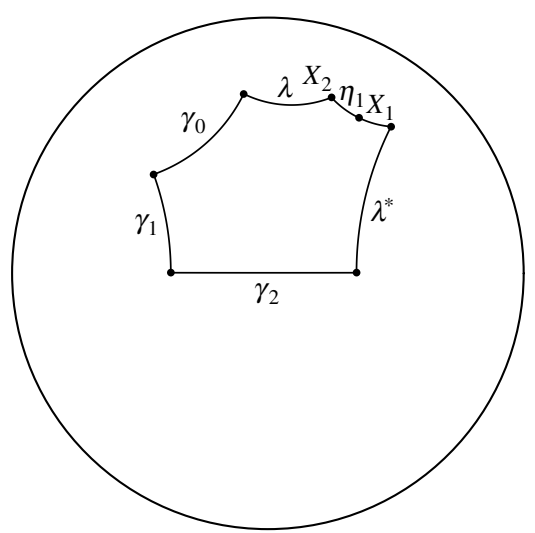

(a)

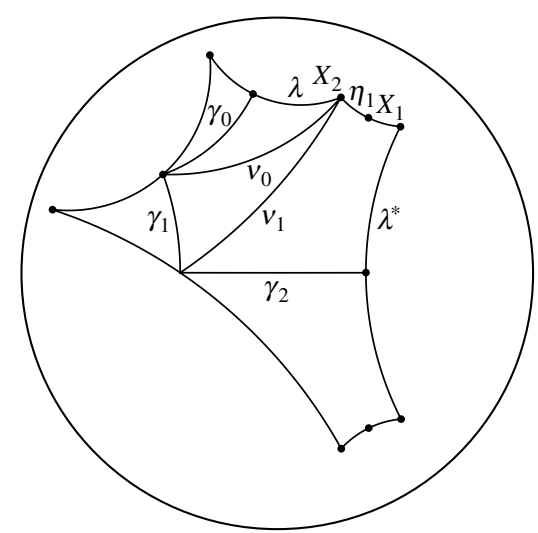

(b)

FIGURE 4

In the obtained region we perform the following procedure: cut by $v_{0}$ and glue $c_{0}\left(v_{0}\right) \rightarrow c_{1}\left(v_{0}\right)$ by means of $x_{3}^{-1}=x_{3}$. From the point $X_{2}$ the perimeter of the region runs now:

$$
X_{2}, v_{0}, X_{3}, x_{3}\left(v_{0}\right), x_{3}\left(X_{2}\right), x_{3}\left(\lambda \cup c_{0}(\lambda)\right), x_{3}\left(c_{0}\left(X_{2}\right)\right), c_{1}\left(v_{1}\right), X_{4}, \ldots
$$

where the angles at the vertices $x_{3}\left(X_{2}\right)$ and $x_{3}\left(c_{0}\left(X_{2}\right)\right)$ are $\phi_{0}$ and $\phi_{0}+\phi_{1}$, respectively.

Let us cut by $\gamma_{1}$ and glue $c_{1}\left(v_{1}\right) \rightarrow c_{2}\left(v_{1}\right)$ by means of $x_{4}$. The perimeter from $X_{3}$ is

$$
\begin{aligned}
X_{3}, \gamma_{1}, X_{4}, x_{4}\left(\gamma_{1}\right), x_{4}\left(X_{3}\right), x_{4} \cdot x_{3}\left(v_{0}\right), x_{4} \cdot x_{3}\left(X_{2}\right), \\
x_{4} \cdot x_{3}\left(\lambda \cup c_{0}(\lambda)\right), c_{2}\left(X_{2}\right), c_{2}\left(v_{2}\right), X_{5}, \ldots
\end{aligned}
$$

where the angle in the vertex $c_{2}\left(X_{2}\right)$ is $\phi_{0}+\phi_{1}+\phi_{2}$.

In this point we change the procedure. Instead of cutting by $\gamma_{2}$ we cut by the line $\rho_{1}$ that joins $X_{4}$ with the point $c_{2}\left(X_{1}\right)=X_{5}$ and we glue $c_{2}\left(\eta_{1}\right) \rightarrow c_{2}\left(\eta_{1}^{\prime}\right)$ by means of $x_{5}=c_{2} \cdot x_{1} \cdot c_{2}$. In the next step cut by $\rho_{2}$ joining $X_{5}$ to $c_{2}\left(X_{2}^{\prime}\right)=X_{6}$ and glue $\left(\lambda \cup c_{0}(\lambda)\right) \rightarrow \lambda^{\prime}$ by means of $x_{5} \cdot x_{4} \cdot x_{3}$.

We have obtained the following polygon (Figure 5):

$$
\begin{array}{r}
X_{2}, v_{0}, X_{3}, \gamma_{1}, X_{4}, \rho_{1}, X_{5}, \rho_{2}, X_{6}, x_{6}\left(\rho_{2}\right), x_{6}\left(X_{5}\right), x_{6} \cdot x_{5}\left(\rho_{1}\right), x_{6} \cdot x_{5}\left(X_{4}\right), \\
x_{6} \cdot x_{5} \cdot x_{4}\left(\gamma_{1}\right), x_{6} \cdot x_{5} \cdot x_{4}\left(X_{3}\right), x_{6} \cdot x_{5} \cdot x_{4} \cdot x_{3}\left(v_{0}\right), X_{2}^{\prime}, \eta_{1}^{\prime}, X_{1}, \eta_{1} .
\end{array}
$$

The angles in the polygon are the following, calling for brevity $x_{6}\left(X_{5}\right)=$ 
$X_{5}^{\prime}, x_{6} \cdot x_{5}\left(X_{4}\right)=X_{4}^{\prime}$ and so on:

$$
\begin{array}{ll}
\widehat{X}_{1}=\widehat{X}_{6}=\pi & \\
\widehat{X}_{2}=\phi_{1}+\phi_{2} & \widehat{X_{2}^{\prime}}=\pi-\left(\phi_{1}+\phi_{2}\right) \\
\widehat{X}_{3}=\alpha_{3}<\frac{\pi}{2} & \widehat{X_{3}^{\prime}}=\pi-\alpha_{3} \\
\widehat{X}_{4}=\alpha_{4}>\frac{\pi}{2} & \widehat{X_{4}^{\prime}}=\pi-\alpha_{4} \\
\widehat{X}_{5}=\alpha_{5} & \widehat{X_{5}^{\prime}}=\pi-\alpha_{5}
\end{array}
$$

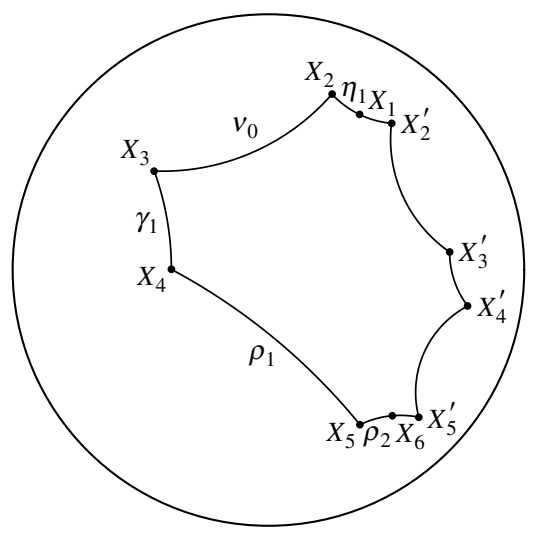

FIGURE 5

For arbitrary $q$ and $s$ we follow the same procedure until arriving to $\gamma_{s}$. Note that the angle in the vertex $c_{s}\left(X_{q}\right)$ is $\phi_{0}+\phi_{1}+\ldots+\phi_{s}=\alpha_{q}$. In this point we cut by the line $\rho_{1}$ that joins $X_{q+s}$ with the point $c_{s}\left(X_{1}\right)$ and we glue $c_{s}\left(\eta_{1}\right) \rightarrow c_{s}\left(\eta_{1}^{\prime}\right)$ by means of $x_{q+s+1}=c_{s} \cdot x_{1} \cdot c_{s}$. In the next step cut by $\rho_{2}$ joining $c_{s}\left(X_{1}\right)$ to $c_{s}\left(X_{2}^{\prime}\right)$ and repeat the process. In each new step $\rho_{j}$ joins $c_{s}\left(X_{j-1}^{\prime}\right)$ to $c_{s}\left(X_{j}^{\prime}\right)$.

Finally the perimeter of the region arrives to be

$$
\begin{aligned}
X_{2}, \eta_{2}, X_{3}, \ldots, X_{q}, v_{0}, X_{q+1}, \gamma_{1}, \ldots, X_{q+s}, & \rho_{1}, X_{q+s+1}, \rho_{2}, \ldots, X_{2}^{\prime}, \eta_{1}^{\prime}, X_{1}, \eta_{1},
\end{aligned}
$$

where the angles are

$$
\begin{aligned}
& \widehat{X}_{1}=\widehat{X}_{2 q+s}=\pi, \\
& \widehat{X}_{i}=\alpha_{i}, \\
& \widehat{X}_{q}=\sum_{i=1}^{s} \phi_{i},
\end{aligned}
$$




$$
\begin{aligned}
\widehat{X}_{q+1} & =\alpha_{q+1}<\frac{\pi}{2}, \\
\widehat{X}_{q+i} & =\alpha_{q+i}=\frac{\pi}{2}, \quad i=2, \ldots, s-1, \\
\widehat{X}_{q+s} & =\alpha_{q+s}>\frac{\pi}{2}, \\
\widehat{X}_{i}^{\prime} & =\pi-\alpha_{i}, \quad i=2, \ldots, q-1, q+1, \ldots, 2 q+s-1, \\
\widehat{X_{q}^{\prime}} & =\pi-\alpha_{q}+\phi_{0}=\pi-\sum_{i=1}^{s} \phi_{i} .
\end{aligned}
$$

- $W_{\Gamma_{3}}$

Let now $W_{\Gamma_{3}}$ be as in Figure 2(b) for an arbitrary $\tau$. Let us reflect the region by means of $c$, the reflection on the side $\gamma$ and then $W_{\Gamma_{3}^{+}}=W_{\Gamma_{3}} \cup c\left(W_{\Gamma_{3}}\right)$.

- $W_{\Gamma_{4}}$

Let now $W_{\Gamma_{4}}$ be as in Figure 3 for an arbitrary $\tau$. Let $W_{\Gamma_{4}^{+}}=W_{\Gamma_{4}} \cup d\left(W_{\Gamma_{4}}\right)$, $d$ being the glide-reflection mapping $\mu^{*} \rightarrow \mu$.

We are ready to establish the following results, for which we remember the above notations: $\alpha_{i}$ and $\alpha_{i}^{\prime}$ are the angles at the vertices $\widehat{X}_{i}$ and $\widehat{X}_{i}^{\prime}$ respectively, and the sides $\eta_{i}$ and $\eta_{i}^{\prime}$ are those between $X_{i}$ and $X_{i+1}$, or $X_{i}^{\prime}$ and $X_{i+1}^{\prime}$, respectively.

THEOREM 4.1. A Fuchsian group $F$ with signature $\left(0,+,\left[2^{2 r}\right],\{-\}\right)$ is the canonical Fuchsian subgroup of an NEC group $\Gamma_{1}$ with signature $(0,+,[-]$, $\left.\left\{\left(2^{r}\right)\right\}\right)$ if $F$ admits a right-angled fundamental region $W_{F}$.

Proof. It is obvious comparing $W_{F}$ with $W_{\Gamma_{1}^{+}}$. In such a case we have:

$$
x_{1}=c_{r} \cdot c_{1}, \quad x_{2}=c_{1} \cdot c_{2}, \quad \ldots, \quad x_{r}=c_{r-1} \cdot c_{r} .
$$

THEOREM 4.2. A Fuchsian group $F$ with signature $\left(0,+,\left[2^{2 r}\right],\{-\}\right)$ is the canonical Fuchsian subgroup of an NEC group $\Gamma_{2}$ with signature $\left(0,+,\left[2^{q}\right]\right.$, $\left.\left\{\left(2^{s}\right)\right\}\right)$, where $2 q+s=2 r$ and $q, s \neq 0$, if $F$ admits a fundamental region $W_{F}$ satisfying the following conditions:

$$
\begin{aligned}
\alpha_{1}=\alpha_{2 r}=\pi, & \\
\alpha_{i}^{\prime}=\pi-\alpha_{i}, & i=2, \ldots, 2 r-1, \\
\eta_{i}^{\prime}=\eta_{i}, & i=1, \ldots, 2 r-1,
\end{aligned}
$$


where besides

$$
\begin{aligned}
\alpha_{q+1} & <\frac{\pi}{2}, \\
\alpha_{q+i} & =\frac{\pi}{2}, \quad i=2, \ldots, s-1, \\
\alpha_{q+s} & >\frac{\pi}{2}, \\
\alpha_{q+s+i} & =\alpha_{i}, \quad i=2, \ldots, q-1, \\
\eta_{q+s+i} & =\eta_{i}, \quad i=1, \ldots, q-1 .
\end{aligned}
$$

Proof. We compare $W_{\Gamma_{2}^{+}}$with $W_{F}$, and we see that $W_{F}$ must satisfy the above conditions.

Let us call

$$
\begin{aligned}
\bar{x}_{i} & =x_{i}, \quad i=1, \ldots, q, \\
\bar{x}_{q+i} & =c_{i-1} \cdot c_{i}, \quad i=1, \ldots, s, \\
\bar{x}_{q+s+1} & =c_{s} \cdot x_{1} \cdot c_{s} \\
\bar{x}_{q+s+2} & =c_{s} \cdot e^{-1} \cdot x_{q} \cdot x_{q-1} \cdot \ldots \cdot x_{3} \cdot x_{2} \cdot x_{3} \cdot \ldots \cdot x_{q-1} \cdot x_{q} \cdot e \cdot c_{s} \\
\bar{x}_{q+s+3} & =c_{s} \cdot e^{-1} \cdot x_{q} \cdot x_{q-1} \cdot \ldots \cdot x_{4} \cdot x_{3} \cdot x_{4} \cdot \ldots \cdot x_{q-1} \cdot x_{q} \cdot e \cdot c_{s} \\
& \vdots \\
\bar{x}_{q+s+q-1} & =c_{s} \cdot e^{-1} \cdot x_{q} \cdot x_{q-1} \cdot x_{q} \cdot e \cdot c_{s} \\
\bar{x}_{q+s+q} & =c_{s} \cdot e^{-1} \cdot x_{q} \cdot e \cdot c_{s}
\end{aligned}
$$

Now we check the product of these elliptic elements:

$$
\begin{aligned}
\prod_{i=1}^{2 q+s} \bar{x}_{i} & =\prod_{i=1}^{q} \bar{x}_{i} \cdot \prod_{i=q+1}^{q+s} \bar{x}_{i} \cdot \prod_{i=q+s+1}^{2 q+s} \bar{x}_{i} \\
& =\prod_{i=1}^{q} x_{i} \cdot \prod_{i=1}^{s}\left(c_{i-1} \cdot c_{i}\right) \cdot\left(\left(c_{s} \cdot x_{1} \cdot c_{s}\right) \cdot \ldots \cdot\left(c_{s} \cdot e^{-1} \cdot x_{q} \cdot e \cdot c_{s}\right)\right) \\
& =\prod_{i=1}^{q} x_{i} \cdot c_{0} \cdot x_{1} \cdot e^{-1} \cdot x_{q} \cdot \ldots \cdot x_{3} \cdot x_{2} \cdot e \cdot c_{s} \\
& =x_{1} \cdot \ldots \cdot x_{q} \cdot c_{0} \cdot e \cdot c_{s} \\
& =x_{1} \cdot \ldots \cdot x_{q} \cdot e \\
& =1
\end{aligned}
$$

THEOREM 4.3. A Fuchsian group $F$ with signature $\left(0,+,\left[2^{2 r}\right],\{-\}\right)$ is the canonical Fuchsian subgroup of an NEC group $\Gamma_{3}$ with signature $\left(0,+,\left[2^{r}\right]\right.$, 
$\{(-)\})$, if $F$ admits a fundamental region $W_{F}$ satisfying the following conditions:

$$
\begin{aligned}
\alpha_{1} & =\alpha_{2 r}=\pi, & & \\
\alpha_{i}^{\prime} & =\pi-\alpha_{i}, & i & =2, \ldots, 2 r-1, \\
\alpha_{i} & =\alpha_{2 r-i+1}, & i & =2, \ldots, r, \\
\eta_{i} & =\eta_{i}^{\prime}, & i & =1, \ldots, r-1, \\
\eta_{i} & =\eta_{2 r-i}, & i & =2, \ldots, r-1 .
\end{aligned}
$$

Proof. In this case we compare $W_{F}$ with $W_{\Gamma_{3}^{+}}$and we see that $W_{F}$ must satisfy the above conditions. In this situation we call

$$
\begin{array}{ll}
\bar{x}_{i}=x_{i}, & i=1, \ldots, \frac{r}{2}, \\
\bar{x}_{i}=c \cdot x_{r-i+1} \cdot c, & i=\frac{r}{2}+1, \ldots, r .
\end{array}
$$

Finally we need only to realize that

$$
\begin{aligned}
\prod_{i=1}^{r} \bar{x}_{i} & =\prod_{i=1}^{\frac{r}{2}} x_{i} \cdot \prod_{i=1}^{\frac{r}{2}} c \cdot x_{r-i+1} \cdot c \\
& =\left(x_{1} \cdot x_{2} \cdot \ldots \cdot x_{\frac{r}{2}}\right) \cdot c \cdot\left(x_{\frac{r}{2}} \cdot \ldots \cdot x_{2} \cdot x_{1}\right) \cdot c \\
& =\left(x_{1} \cdot x_{2} \cdot \ldots \cdot x_{\frac{r}{2}}\right) \cdot c \cdot e \cdot c \\
& =1 .
\end{aligned}
$$

THEOREM 4.4. A Fuchsian group $F$ with signature $\left(0,+,\left[2^{2 r}\right],\{-\}\right)$ is the canonical Fuchsian subgroup of an NEC group $\Gamma_{4}$ with signature $\left(1,-,\left[2^{r}\right]\right.$, $\{-\})$, if $F$ admits a fundamental region $W_{F}$ satisfying the following conditions:

$$
\begin{array}{rlrl}
\alpha_{1} & =\alpha_{2 r}=\pi, & \\
\alpha_{i}^{\prime}=\pi-\alpha_{i}, & i=2, \ldots, 2 r-1, \\
\alpha_{i}=\alpha_{2 r-i+1}, & i=2, \ldots, r-1, \\
\alpha_{r}>\alpha_{r+1}, & \\
\eta_{i}^{\prime}=\eta_{i}, & i=1, \ldots, 2 r-1, \\
\eta_{i}=\eta_{2 r-i}, & i=2, \ldots, r-1,
\end{array}
$$

Proof. By comparing $W_{F}$ with $W_{\Gamma_{4}^{+}}$we see that $W_{F}$ must satisfy the above 
conditions. Call

$$
\begin{array}{ll}
\bar{x}_{i}=x_{i}, & i=1, \ldots, \frac{r}{2}, \\
\bar{x}_{i}=d \cdot x_{r-i+1} \cdot d^{-1}, & i=1, \ldots, \frac{r}{2} .
\end{array}
$$

At last, we check that

$$
\begin{aligned}
\prod_{i=1}^{r} \bar{x}_{i} & =\prod_{i=1}^{\frac{r}{2}} x_{i} \cdot \prod_{i=1}^{\frac{r}{2}}\left(d \cdot x_{r-i+1} \cdot d^{-1}\right) \\
& =\left(x_{1} \cdot x_{2} \cdot \ldots \cdot x_{\frac{r}{2}}\right) \cdot d \cdot\left(x_{\frac{r}{2}} \cdot \ldots \cdot x_{2} \cdot x_{1}\right) \cdot d^{-1} \\
& =\left(x_{1} \cdot x_{2} \cdot \ldots \cdot x_{\frac{r}{2}}\right) \cdot d \cdot d^{2} \cdot d^{-1} \\
& =1 .
\end{aligned}
$$

\section{Hyperelliptic surfaces}

In this section we characterize the hyperelliptic and symmetric Riemann surfaces by means of the results of Section 4 about fundamental regions. Previously we need the following results about hyperelliptic Klein surfaces.

Let $X=\mathscr{D} / \Gamma^{*}$ be a Klein surface where $\Gamma^{*}$ has a signature $(g ; \pm ;[-]$, $\left.\left\{(-)^{k}\right\}\right)$. The surface $X$ is hyperelliptic if and only if there exists an NEC group $\Gamma$ containing $\Gamma^{*}$ as a subgroup of index 2 having one of the following signatures:

If $k \neq 0,[2$, Theorem 4.5]:
i) $\left(0,+,[-],\left\{\left(2^{2 k}\right)\right\}\right) \quad g=0, \quad k \geq 3$.
ii) $\left(0,+,\left[2^{g+k}\right],\{(-)\}\right) \quad g>0, \quad “+”, \quad k=1,2$.
iii) $\left(0,+,\left[2^{g}\right],\left\{\left(2^{2 k}\right)\right\}\right) \quad g>0, \quad$ “”, $\quad k>0$.

If $k=0$, and in this case $X$ must be a nonorientable unbordered surface (see [5, Theorem 2.2] for the case $q=0$ ):

iv) $\left(0,+,\left[2^{g}\right],\{(-)\}\right) \quad g \geq 3, \quad$ “",$\quad k=0$.

v) $\left(1,-,\left[2^{g}\right],\{-\}\right) \quad g>3, \quad$ “", $\quad k=0, \quad g$ even.

Let $S=\mathscr{D} / F^{*}$ be a hyperelliptic and symmetric Riemann surface of genus $p \geq 2$. Since $S$ is hyperelliptic there exists a Fuchsian group $F$ containing $F^{*}$ as a subgroup of index 2 , with signature $\left(0,+,\left[2^{2 p+2}\right],\{-\}\right)$, and because $S$ is symmetric, with a symmetry $\psi$, then $S /\langle\psi\rangle=X=\mathscr{D} / \Gamma^{*}$, where $X$ is a hyperelliptic Klein surface and so there exists an NEC group $\Gamma$ with one of the 
five above signatures. Moreover, the group $F$ must be the Fuchsian canonical subgroup of the NEC group $\Gamma$ and we may apply the geometric results on the respective fundamental regions of $F$ and $\Gamma^{+}$. With the above notations, we rewrite without proofs the Theorems 4.1-4.4 in terms of surfaces, where $S=$ $\mathscr{D} / F^{*}$ is a given hyperelliptic Riemann surface and $F$ is the hyperellipticity group, and $W_{\Gamma_{i}^{+}}$denotes the corresponding regions in Theorems 4.1-4.4.

THEOREM 5.1. If $F$ admits a fundamental region $W_{F}=W_{\Gamma_{1}^{+}}$then $S$ has an automorphism $\psi$ such that $X=S /\langle\psi\rangle$ is a orientable hyperelliptic Klein surface with topological genus $g=0$, and $k=p+1$ boundary components.

THEOREM 5.2. If $F$ admits a fundamental region $W_{F}=W_{\Gamma_{2}^{+}}$then $S$ has an automorphism $\psi$ such that $X=S /\langle\psi\rangle$ is a non-orientable hyperelliptic Klein surface with topological genus $g$, and $k>0$ boundary components, where $g+k=p$.

THEOREM 5.3. If $F$ admits a fundamental region $W_{F}=W_{\Gamma_{3}^{+}}$then $S$ has an automorphism $\psi$ such that $X=S /\langle\psi\rangle$ is either

- an orientable hyperelliptic Klein surface with topological genus g, and $k=1$ or 2 boundary components, where $g+k=p+1$; or

- a non-orientable hyperelliptic unbordered Klein surface with topological genus $g=p+1$.

THEOREM 5.4. If $F$ admits a fundamental region $W_{F}=W_{\Gamma_{4}^{+}}$then $S$ has an automorphism $\psi$ such that $X=S /\langle\psi\rangle$ is a non-orientable unbordered hyperelliptic Klein surface with topological genus $g=p+1$. In this case $p$ must be odd.

Let us note that $q$-hyperelliptic surfaces are too defined by groups whose signatures have all periods equal to $2,[1]$ and [3]. We feel that similar procedures to the above ones, applied to the fundamental regions of these groups also should work. To do that, it shall be necessary to study the fundamental regions of NEC groups with several empty cycle-periods.

AcKnowledgements. We wish to thank Professor E. Bujalance for several helpful comments as well as the referee for helpful suggestions.

\section{REFERENCES}

1. Bujalance, E., Etayo, J. J., Characterization of q-Hyperelliptic compact planar Klein surfaces, Abh. Math. Sem. Univ. Hamburg 58 (1988), 95-104.

2. Bujalance, E., Etayo, J. J., Gamboa, J. M., Hyperelliptic Klein surfaces, Quart. J. Math. Oxford (2) 36 (1985), 141-157.

3. Bujalance, E., Etayo, J. J., Gamboa, J. M., Superficies de Klein elípticas hiperelípticas, Memorias de la Real Academia de Ciencias, Tomo XIX (1985). 
4. Bujalance, E., Singerman, D., The symmetry type of a Riemann surface, Proc. London Math. Soc. (3) 51 (1985), 501-519.

5. Bujalance, J. A., Hyperelliptic compact non-orientable Klein surfaces without boundary, Kodai Math. J. 12 (1989), 1-8.

6. Maskit, B., A New Characterization of Hyperellipticity, Michigan Math. J. 47 (2000), 3-14.

7. Macbeath, A. M., The classification of non-euclidean crystallographic groups, Canad. J. Math. 19 (1967), 1192-1205.

8. Schmutz Schaller, P., Geometric characterization of hyperelliptic Riemann surfaces, Ann. Acad. Sci. Fenn. Math. 25 (2000), 85-90.

9. Singerman, D., On the structure of non-euclidean crystallographic groups, Proc. Cambridge Philos. Soc. 76 (1974), 233-240.

10. Wilkie, H. C., On non-Euclidean crystallographic groups, Math. Z. 91 (1966), 87-102.

11. Wiman, A., Über die hyperelliptischen Kurven und diejenigen vom Geschlecht $p=3$, welche eindeutigen Transformationen in sich zulassen, Bihang Till. Kongl. Svenska VetenskapsAkademiens Handlingar 21,1 n.1 (1895), 23 pp.

DEPARTAMENTO DE ÁLGEBRA

FACULTAD DE CIENCIAS MATEMÁTICAS

UNIVERSIDAD COMPLUTENSE

28040-MADRID

SPAIN

E-mail: jetayo@mat.ucm.es
DEPARTAMENTO DE MATEMÁTICAS FUNDAMENTALES

FACULTAD DE CIENCIAS

UNIVERSIDAD NACIONAL DE EDUCACIÓN A DISTANCIA 28040-MADRID

SPAIN

E-mail: emartinez@mat.uned.es 